\title{
Comparative Study between Hysterosalpingography, Saline Infusion Transvaginal Ultrasound and Doppler Studies in Assessment of Uterine Factor of Infertility
}

\author{
Mohammed Hesham Hassan Anwar, Osama Elsaeid Ali, Bassem Ragab Abdelaziz and Mohammed \\ Abdalla Hassanin * \\ Obstetrics and Gynaecology Department, Faculty of Medicine, Al Azhar University \\ *Corresponding Author: Mohammed Abdalla Hassanin,Phone No.: (+2) 01156669577,E-mail: \\ mmoheyeldien@gmail.com
}

\begin{abstract}
Background: One of the basic steps of an infertility workup is to evaluate the shape and regularity of the uterine cavity. Acquired uterine lesions, such as uterine fibroids, endometrial polyps, intrauterine adhesions, or all of these, may cause infertility by interfering with proper embryo implantation and growth.
\end{abstract}

Aim of the Work: to compare the findings of hysterosalpingography, saline infusion transvaginal sonohysterography and Doppler studies in patients with suspected uterine causes of infertility. Patients and Methods: This is a comparative observational cross sectional study, which was conducted in the Obstetrics and Gynecology Department at Al Hussein and Sayed Galal University Hospitals, Al Azhar University in Cairo during the period from April 2017 to the end of September 2018. Sixty patients were included at the study who were recruited from the infertility outpatient clinics, with suspected uterine factors of infertility clinically, by hysterosalpingography (HSG) or by ultrasound. Results: HSG showed the highest sensitivity in the diagnosis of bicornuate uterus, septate uterus and intrauterine synechia (100\%) followed by endometrial polyp (70\%) then submucous myomas $(62.5 \%)$. High specificity (100\%) was in the diagnosis of endometrial polyp and submucous myoma. Also it showed high false negative values as it missed the diagnosis of 7 cases.

Conclusion: SIS is the gold standard of our study in assessment of uterine factor of infertility. Doppler studies showed high false negative results compared to HSG and saline infusion sonohysterography (SIS). Some lesions were missed from HSG and diagnosed by SIS and these missed lesions may play a role in infertility, so SIS is very important modality in diagnosis and assessment of uterine factor of infertility.

Keywords: Hysterosalpingography - Saline Infusion Transvaginal Ultrasound - Doppler

\section{INTRODUCTION}

In fact, infertility related to uterine cavity abnormalities has been estimated to be the causal factor in as many as $10 \%$ to $15 \%$ of couples seeking treatment. Moreover, abnormal uterine findings have been found in $34 \%$ to $62 \%$ of infertile women ${ }^{(\mathbf{1})}$.

Traditionally hysterosalpingography (HSG) has been the most commonly used technique in the evaluation of infertility. It gives reliable information about the patency and morphology of the fallopian tubes. It is also helpful in evaluating uterine cavity abnormalities (2). Sonohysterography ( $\mathrm{SHG}$ ) is considered a simple, effective and well tolerated technique for enhanced transvaginal sonographic imaging of the endometrial cavity. The instillation of sterile saline into the uterine cavity via a fine catheter provides both a contrast medium and an expanding agent. So, saline infusion sonohysterography can help to triage patients to (1) no anatomic pathology, (2) globally thickened anatomic pathology that may be evaluated with blind endometrial sampling, or (3) focal abnormalities that must be evaluated under direct vision ${ }^{(3)}$.

Transvaginal pulsed wave (PW) Doppler ultrasound is an important tool for examining the female reproductive system and is a noninvasive method to assess the uterine perfusion. However blood flow characteristics within the uterine artery may not be representative of endometrial perfusion ${ }^{(3)}$.

PW Doppler may be used to examine the smaller downstream radial and spiral arteries; it reveals information from single vessels rather than from the endometrium as a whole. 
Colour Doppler (CD) ultrasound may be used to examine the uterine vasculature as a whole through demonstration of blood flow as a colour map. Subjective analysis of vessel distribution has shown that the absence of subendometrial and intra-endometrial $\mathrm{CD}$ signals is associated with non conception cycles (1).

Power Doppler (PD) is better suited to the study of endometrial perfusion as it is more sensitive to low flow and thus overcomes the problems of angle dependence and background noise associated with both CD and PW Doppler (4).

$\mathrm{CD}$ provides qualitative information. The Power Doppler (PW) signal can be subsequently analyzed to produce quantitative information through one of several computer software packages ${ }^{(5)}$.

In combination with three-dimensional (3D) ultrasound the PD offers tool with which one may not only demonstrate but also quantify total endometrial and regional uterine blood flow ${ }^{(\boldsymbol{6})}$.

\section{AIM OF THE WORK}

The aim of this work is to compare the findings of hysterosalpingography, saline infusion transvaginal sonohysterography and Doppler studies in patients with suspected uterine causes of infertility.

\section{PATIENTS AND METHODS}

A comparative observational cross sectional study was conducted in the Obstetrics and Gynecology Department at Al Hussein and Sayed Galal University Hospitals, Al Azhar University in Cairo during the period from April 2017 to the end of September 2018.

Sixty patients were included in the study who were recruited from the infertility outpatient clinics, with suspected uterine factors of infertility clinically, by HSG or by ultrasound.

\section{Inclusion criteria:}

- Age between 20 and 35 years.

- Primary or secondary infertility.

- Normal semen analysis of their husbands.

- Regular ovulation as evident by regular menstrual cycle, confirmed by transvaginal $\mathrm{U} / \mathrm{S}$ and day 21 serum progesterone.

- Average body mass index (BMI) from 18$25 \mathrm{~kg} \backslash \mathrm{m}^{2}$.

- Positive tubal patency as evaluated in HSG.

Exclusion criteria:
Patients who were excluded from the study were those having any contraindications for sonohysterography (menstruation, pregnancy, severe vaginitis or cervicitis, endometrial infection, history of recent pelvic inflammatory diseases or vaginal bleeding).

All the patients participating were informed and consented for the study and were subjected to the following:

1. Personal history: name, age, parity, occupation, residency and special habits.

2. Husband history: name, age, occupation, residency, special habits and diseases.

3. Sexual history: coital frequency, timing in relation to the cycle, use of vaginal lubricant before or vaginal douching after coitus as well as any associated problem as difficult or painful coitus.

4. Past history: medical diseases, abdominal surgeries, drug therapy or allergy.

5. Menstrual history: menarche, regularity, duration, amount and associated pain.

6. General and local examination.

7. Routine infertility investigations and postmenstrual hormonal profile were done or reevaluated if already done and were being within normal as husband semen analysis, FSH, LH, prolactin, TSH and midluteal progesterone and serum free Testosterone.

8. Postmenstrual transvaginal ultrasound.

9. Postmenstrual hysterosalpingography using water soluble dye was done.

10. Sonohysterography using saline infusion with pediatrics' Foley's catheter size ${ }^{(6)}$.

11. TVS colour Doppler was done for measurements of radial and spiral artery impedance and PI value was calculated.

\section{Statistical analysis}

Results were expressed as means \pm standard deviation (SD) or number (\%). Comparison between categorical data was performed using Chi square test. Standard diagnostic indices including sensitivity, specificity, positive predictive value (PPV), negative predictive value (NPV) and diagnostic accuracy were calculated. The data were considered significant if $p$ values was $\leq 0.05$ and highly significant if $p<0.01$. Statistical analysis was performed with the aid of the SPSS computer program (version 25 IBM 2015). 
Table (1): Demographic features of the studied cases

\begin{tabular}{|l|l|l|l|l|l|}
\hline & N & Minimum & Maximum & Mean & Std. Deviation \\
\hline Age & 60 & 21.00 & 35.00 & 28.70 & 4.88 \\
\hline Married for (yrs.) & 60 & 2.00 & 13.00 & 5.65 & 3.76 \\
\hline Period of infertility (yrs.) & 60 & 2.00 & 8.00 & 3.94 & 1.87 \\
\hline
\end{tabular}

Mean age of patients was; 27.6 years, SD 4.6 (range; 21-35). Mean duration of infertility was; 3.79 years, SD 1.8 (range; 2 - 8).

Table (2): Type of infertility of the studied cases

\begin{tabular}{|l|l|l|}
\hline & Number & Percent \\
\hline 1ry & 32 & $53.3 \%$ \\
\hline 2ry & 28 & $46.7 \%$ \\
\hline Total & 60 & $100 \%$ \\
\hline
\end{tabular}

Thirty nine cases suffered from primary infertility $(65 \%)$, while twenty one cases were suffering from secondary infertility (35\%).

Table (3): Distribution of findings of SIS among the studied cases

\begin{tabular}{|l|l|l|}
\hline & Number & Percent \\
\hline Polyp & 20 & $33.3 \%$ \\
\hline Submucous myoma & 16 & $26.7 \%$ \\
\hline Septate uterus & 7 & $11.7 \%$ \\
\hline Bicornuate uterus & 6 & $10.0 \%$ \\
\hline Intrauterine synechia & 6 & $10.0 \%$ \\
\hline Free (NAD) & 5 & $8.3 \%$ \\
\hline
\end{tabular}

Table (4) shows the number and percentage of different findings among the studied cases as diagnosed by the gold standard SIS. The most common abnormality was endometrial polyp with percentage of $33.3 \%$.

Table (4): Comparison between positive and negative findings of SIS

\begin{tabular}{|l|l|l|}
\hline & Number & Percent \\
\hline Negative & 5 & $8.3 \%$ \\
\hline Positive & 55 & $91.7 \%$ \\
\hline
\end{tabular}

55 patients $(91.7 \%)$ had positive findings by SIS while 5 patients $(8.3 \%)$ had no findings.

Table (5): Distribution of the findings of HSG among the studied cases

\begin{tabular}{|l|l|l|}
\hline \multicolumn{1}{|c|}{ Number } & Percent \\
\hline Polyp & 14 & $23.3 \%$ \\
\hline Myoma & 10 & $16.7 \%$ \\
\hline Septate uterus & 10 & $16.7 \%$ \\
\hline Bicornuate uterus & 8 & $13.3 \%$ \\
\hline Intrauterine synechia & 9 & $15.0 \%$ \\
\hline Free (NAD) & 9 & $15.0 \%$ \\
\hline
\end{tabular}

With hysterosalpingography, 51 abnormalities $(85 \%)$ were suspected. The most common pathology was endometrial polyp (14 cases, $23.3 \%)$.

Table (6): Description of the duration of infertility among the findings of SIS of the studied cases

\begin{tabular}{|l|l|l|l|}
\hline & Number & Mean & Std. Deviation \\
\hline Polyp & 20 & 3.64 & 1.76 \\
\hline Submucous myoma & 16 & 4.26 & 2.39 \\
\hline Septate uterus & 7 & 4.86 & 2.34 \\
\hline Bicornuate uterus & 6 & 3.12 & 2.04 \\
\hline Intrauterine synechia & 6 & 3.32 & 1.66 \\
\hline Free (NAD) & 5 & 4.04 & 1.80 \\
\hline
\end{tabular}

Duration of infertility among findings of SIS of the studied cases revealed that patients with endometrial polyp have about 3.64 years of infertility, submucous fibroid 4.26 years, septate uterus 4.86 years, bicornuate uterus 3.12 years and intrauterine synechia 3.32 years of infertility.

Table (7): Description of Age among different findings of SIS of the studied cases 


\begin{tabular}{|l|l|l|l|}
\hline & Number & Mean & Std. Deviation \\
\hline Polyp & 20 & 26.66 & 4.71 \\
\hline Submucous myoma & 16 & 31.20 & 2.94 \\
\hline Septate uterus & 7 & 28.60 & 4.95 \\
\hline Bicornuate uterus & 6 & 21.84 & 3.75 \\
\hline Intrauterine synechia & 6 & 28.48 & 6.03 \\
\hline Free (NAD) & 5 & 29.82 & 4.60 \\
\hline
\end{tabular}

Table (8): Description of the type of infertility among the findings of SIS of the studied cases

\begin{tabular}{|l|l|l|l|l|}
\hline \multirow{2}{*}{ Polyp } & 1ry $(\mathbf{n}=\mathbf{3 2})$ & 2ry $(\mathbf{n}=\mathbf{2 8})$ \\
\cline { 2 - 5 } & Number & Percent & Number & Percent \\
\hline Submucous myoma & 13 & $40.6 \%$ & 7 & $25.0 \%$ \\
\hline Septate uterus & 6 & $18.8 \%$ & 10 & $35.7 \%$ \\
\hline Bicornuate uterus & 6 & $18.8 \%$ & 1 & $3.6 \%$ \\
\hline Intrauterine synechia & 4 & $12.5 \%$ & 2 & $7.1 \%$ \\
\hline Free (NAD) & 0 & $0.0 \%$ & 6 & $21.4 \%$ \\
\hline p-value & 3 & $9.4 \%$ & 2 & $7.1 \%$ \\
\hline
\end{tabular}

Table (9): Comparison between positive and negative findings of HSG

\begin{tabular}{|l|l|l|}
\hline & Number & Percent \\
\hline Negative & 9 & $15.0 \%$ \\
\hline Positive & 51 & $85.0 \%$ \\
\hline
\end{tabular}

51 patients (85\%) have positive findings by HSG while 9 patients (15\%) have no findings.

Table (10): Distribution of the findings of Doppler among the studied cases

\begin{tabular}{|l|l|l|}
\hline & Number & Percent \\
\hline Polyp & 9 & $15.0 \%$ \\
\hline Submucous myoma & 12 & $20.0 \%$ \\
\hline Septate uterus & 6 & $10.0 \%$ \\
\hline Bicornuate uterus & 10 & $16.7 \%$ \\
\hline Intrauterine synechia & 5 & $8.3 \%$ \\
\hline Free (NAD) & 18 & $30.0 \%$ \\
\hline
\end{tabular}

With Doppler studies, 42 abnormalities (70\%) were suspected, with the most common pathology was submucous myoma, 12 cases (20\%).

Table (11): Comparison between positive and negative findings of Doppler studies

\begin{tabular}{|l|l|l|}
\hline & Number & Percent \\
\hline Negative & 18 & $30.0 \%$ \\
\hline Positive & 42 & $70.0 \%$ \\
\hline
\end{tabular}

With Doppler studies on studied cases 42 patients (70\%) have positive findings while 18 patients (18\%) have no findings.

Table (12): Findings of different used techniques among all the studied cases

\begin{tabular}{|c|c|c|c|c|c|c|}
\hline & \multicolumn{2}{|l|}{ SIS } & \multicolumn{2}{|c|}{ HSG } & \multicolumn{2}{|c|}{ Doppler } \\
\hline & No. & $\%$ & No. & $\%$ & No. & $\%$ \\
\hline Polyp & 20 & $33.3 \%$ & 14 & $23.3 \%$ & 9 & $15.0 \%$ \\
\hline Submucous myoma & 16 & $26.7 \%$ & 10 & $16.7 \%$ & 12 & $20.0 \%$ \\
\hline Septate uterus & 7 & $11.7 \%$ & 10 & $16.7 \%$ & 6 & $10.0 \%$ \\
\hline Bicornuate uterus & 6 & $10.0 \%$ & 8 & $13.3 \%$ & 10 & $16.7 \%$ \\
\hline Intrauterine synechia & 6 & $10.0 \%$ & 9 & $15.0 \%$ & 5 & $8.3 \%$ \\
\hline Free (NAD) & 5 & $8.3 \%$ & 9 & $15.0 \%$ & 18 & $30.0 \%$ \\
\hline
\end{tabular}

Table (13): Comparison between positive findings of HSG and SIS among the studied cases

\begin{tabular}{|c|c|c|c|}
\hline \multirow{2}{*}{ HSG } & \multicolumn{2}{|l|}{ SIS } & \multirow{2}{*}{$P$ value } \\
\hline & Negative $(n=5)$ & Positive $(n=55)$ & \\
\hline Negative $(n=9)$ & $2(40 \%)$ & $\begin{array}{ll}7 & (12.7 \%) \\
\end{array}$ & \multirow{2}{*}{$>0.05$} \\
\hline Positive $(n=51)$ & $3(60 \%)$ & $48(87.3 \%)$ & \\
\hline
\end{tabular}

Data are expressed as number (\%).

$\mathrm{NS}=\mathrm{p}>0.05=$ not significant . 
When comparing the results HSG with that of the gold standard SIS, we found that out of 9 cases that were diagnosed by HSG as free of abnormalities, only 2 were confirmed with SIS, the rest had different pathologies that were missed by the HSG. And out of 51 cases that were diagnosed by HSG as abnormal finding, only 48 cases were confirmed by SIS.

Table (14): Comparison between positive findings of Doppler studies and SIS among the studied cases

\begin{tabular}{|l|l|l|l|}
\multirow{2}{*}{ Doppler } & SIS & \multirow{2}{*}{ P value } \\
\cline { 2 - 3 } & Negative $(\mathbf{n}=\mathbf{5})$ & Positive $(\mathbf{n}=\mathbf{5 5})$ & \multirow{2}{*}{0.05} \\
\hline Negative $(\mathbf{n}=\mathbf{1 8})$ & $3(60 \%)$ & $15(27.3 \%)$ & $>0.05$ \\
\hline Positive $(\mathbf{n}=\mathbf{4 2})$ & $2(40 \%)$ & $40(72.7 \%)$ & \\
\hline
\end{tabular}

Data are expressed as number (\%). NS=p $>0.05=$ not significant.

When comparing the results of Doppler studies with that of the gold standard SIS, we found that out of 18 cases that were diagnosed by Doppler studies as free of abnormalities, only 3 were confirmed with SIS, the rest had different pathologies that were missed by Doppler studies. And out of 42 cases that were diagnosed by Doppler studies as abnormal finding, only 40 cases were confirmed by SIS.

Table (15): Sensitivity, specificity, PPV, NPV, and accuracy of different used techniques in the diseased cases compared to SIS.

\begin{tabular}{|l|l|l|l|l|l|}
\hline & Sensitivity & Specificity & PPV & NPV & Accuracy \\
\hline \multirow{2}{*}{ Doppler } & $40 / 55$ & $3 / 5$ & $40 / 42$ & $3 / 18$ & $43 / 60$ \\
& $(72.7 \%)$ & $(60 \%)$ & $(95.2 \%)$ & $(16.7 \%)$ & $(71.7 \%)$ \\
\hline \multirow{2}{*}{ HSG } & $48 / 55$ & $2 / 5$ & $48 / 51$ & $2 / 9$ & $50 / 60$ \\
& $(87.3 \%)$ & $(40 \%)$ & $(94.1 \%)$ & $(22.2 \%)$ & $(83.3 \%)$ \\
\hline
\end{tabular}

From the previous data, sensitivity, specificity, positive predictive value, negative predictive value \& accuracy of both HSG \& Doppler studies are calculated and compared to the gold standard SIS. This table showed that HSG had higher values than Doppler studies in the most of different parameters.

Table (16): Sensitivity, specificity, PPV, NPV, and accuracy of HSG for different findings compared to SIS

\begin{tabular}{|l|l|l|l|l|l|}
\hline & Sensitivity & Specificity & PPV & NPV & Accuracy \\
\hline \multirow{2}{*}{ Polyp } & $14 / 20$ & $40 / 40$ & $14 / 14$ & $40 / 46$ & $54 / 60$ \\
& $(70.0 \%)$ & $(100.0 \%)$ & $(100.0 \%)$ & $(87.0 \%)$ & $(90.0 \%)$ \\
\hline \multirow{2}{*}{ Submucous myoma } & $10 / 16$ & $44 / 44$ & $10 / 10$ & $44 / 50$ & $54 / 60$ \\
& $(62.5 \%)$ & $(100.0 \%)$ & $(100.0 \%)$ & $(88.0 \%)$ & $(90.0 \%)$ \\
\hline \multirow{2}{*}{ Septate uterus } & $7 / 7$ & $50 / 53$ & $7 / 10$ & $50 / 50$ & $57 / 60$ \\
& $(100.0 \%)$ & $(94.3 \%)$ & $(70.0 \%)$ & $(100.0 \%)$ & $(95.0 \%)$ \\
\hline \multirow{2}{*}{ Bicornuate uterus } & $6 / 6$ & $52 / 54$ & $6 / 8$ & $52 / 52$ & $58 / 60$ \\
& $(100.0 \%)$ & $(96.3 \%)$ & $(75.0 \%)$ & $(100.0 \%)$ & $(96.7 \%)$ \\
\hline \multirow{2}{*}{ Intrauterine synechia } & $6 / 6$ & $51 / 54$ & $6 / 9$ & $51 / 51$ & $57 / 60$ \\
& $(100.0 \%)$ & $(94.4 \%)$ & $(66.7 \%)$ & $(100.0 \%)$ & $(95.0 \%)$ \\
\hline \multirow{2}{*}{ Free (NAD) } & $5 / 5$ & $51 / 55$ & $5 / 9$ & $51 / 51$ & $56 / 60$ \\
& $(100.0 \%)$ & $(92.7 \%)$ & $(55.6 \%)$ & $(100.0 \%)$ & $(93.3 \%)$ \\
\hline
\end{tabular}

Table (17): Sensitivity, specificity, PPV, NPV, and accuracy of Doppler studies for different findings compared to SIS

\begin{tabular}{|l|l|l|l|l|l|}
\hline & Sensitivity & Specificity & PPV & NPV & Accuracy \\
\hline \multirow{2}{*}{ Polyp } & $9 / 20$ & $40 / 40$ & $9 / 9$ & $40 / 51$ & $49 / 60$ \\
& $(45.0 \%)$ & $(100.0 \%)$ & $(100.0 \%)$ & $(78.4 \%)$ & $(81.7 \%)$ \\
\hline \multirow{2}{*}{ Submucous myoma } & $12 / 16$ & $44 / 44$ & $12 / 12$ & $44 / 48$ & $56 / 60$ \\
& $(75.0 \%)$ & $(100.0 \%)$ & $(100.0 \%)$ & $(91.7 \%)$ & $(93.3 \%)$ \\
\hline \multirow{2}{*}{ Septate uterus } & $6 / 7$ & $53 / 53$ & $6 / 6$ & $53 / 54$ & $59 / 60$ \\
& $(85.7 \%)$ & $(100.0 \%)$ & $(100.0 \%)$ & $(98.1 \%)$ & $(98.3 \%)$ \\
\hline \multirow{2}{*}{ Bicornuate uterus } & $6 / 6$ & $50 / 54$ & $6 / 10$ & $50 / 50$ & $56 / 60$ \\
& $(100.0 \%)$ & $(92.6 \%)$ & $(60.0 \%)$ & $(100.0 \%)$ & $(93.3 \%)$ \\
\hline \multirow{2}{*}{ Intrauterine synechia } & $5 / 6$ & $54 / 54$ & $5 / 5$ & $54 / 55$ & $59 / 60$ \\
& $(83.3 \%)$ & $(100.0 \%)$ & $(100.0 \%)$ & $(98.2 \%)$ & $(98.3 \%)$ \\
\hline \multirow{2}{*}{ Free (NAD) } & $9 / 18$ & $42 / 42$ & $9 / 9$ & $42 / 51$ & $51 / 60$ \\
$(85.0 \%)$
\end{tabular}


Comparative Study between Hysterosalpingography, Saline Infusion Transvaginal...

Table (18): Findings detected by different diagnostic techniques compared to SIS

\begin{tabular}{|l|l|l|l|}
\hline & HSG & Doppler & SIS \\
\hline Polyp & $14 / 20(70.0 \%)$ & $9 / 20(45.0 \%)$ & 20 \\
\hline Submucous myoma & $10 / 16(62.5 \%)$ & $12 / 16(75.0 \%)$ & 16 \\
\hline Septate uterus & $7 / 7(100.0 \%)$ & $6 / 7(85.7 \%)$ & 7 \\
\hline Bicornuate uterus & $6 / 6(100.0 \%)$ & $6 / 6(100.0 \%)$ & 6 \\
\hline Intrauterine synechia & $6 / 6(100.0 \%)$ & $5 / 6(83.3 \%)$ & 6 \\
\hline Free (NAD) & $5 / 5(100.0 \%)$ & $9 / 18(50.0 \%)$ & 5 \\
\hline Total & $48 / 60$ & $47 / 60$ & 60 \\
\hline
\end{tabular}

From the previous data, SIS was the gold standard of this study to assess the uterine factor of infertility, while HSG was more accurate than Doppler studies for assessment of uterine factor of infertility.

\section{DISCUSSION}

Infertility remains a major clinical and social problem, affecting perhaps one couple in six ${ }^{(7)}$. Worldwide more than 70 million couples suffer from infertility, the majority being residents of developing countries. The exact prevalence of infertility in developing countries is unknown due to a lack of registration and well-performed studies. On the other hand the implementation of appropriate infertility treatment is currently not a main goal for most international non-profit organizations ${ }^{(8)}$.

One of the basic steps of an infertility workup is to evaluate the shape and regularity of the uterine cavity ${ }^{(9)}$.

Acquired uterine lesions, such as uterine fibroids, endometrial polyps, intrauterine adhesions, or all of these, may cause infertility by interfering with proper embryo implantation and growth. Congenital uterine malformations are also thought to play a role in delaying natural conception ${ }^{(\mathbf{1 0})}$.

There are different methods for assessment of uterine factor of infertility as 4D ultrasound, MRI on pelvis and hysteroscopy.

We choose HSG, SIS and Doppler as they are available, cheap for the patients and not invasive and do not require anesthesia.

In our study SIS was the best method to evaluate uterine factor of infertility and should be confirmed by hysteroscopy. Hysteroscopy is the definite method for evaluation of the uterine cavity and diagnosis of associated abnormalities, but it is invasive, more expensive and needs anesthesia ${ }^{(\mathbf{1 1})}$.

In our study, sixty patients with suspected uterine factors of infertility were included, all presenting with infertility. 39 patients suffered from 1ry infertility (65\%), and 21 patients had 2ry infertility (35\%). 2ry infertility included patients who had previous living offsprings or previous abortions with no living children. Intrauterine synechia was only found in cases of 2ry infertility. $65 \%$ of cases of endometrial polyp were in patients with 1ry infertility, the rest were with 2ry infertility. $62.5 \%$ of cases of myomas were with 2ry infertility. While $85.7 \%$ of septate uterus were in cases with 1ry infertility. The age of the patients was limited between 21-35, mean age was 28.7 years (SD 4.88). Myomas were prevalent in higher age groups (mean age 31.2 years, SD 2.94). Duration of infertility ranged from 2 to 8 years (mean 3.94 years, SD 1.87). Longest duration was with septate uterus (mean 4.86 years, SD 2.34), while the least was with bicornuate uterus (mean 3.12 years, SD 2.04).

In this study we found that SIS showed the following results; 5 patient $(8.3 \%)$ were found to have normal uterine cavity and cervix. 55 patients $(91.7 \%)$ showed significant abnormal findings, in the later group the SIS findings included presence of intrauterine polyps, intrauterine synechia, submucous fibroids, uterine septum and bicornuate uterus. 20 patients $(33.3 \%)$ were noted to have intrauterine polyps, 6 patients $(10 \%)$ were discovered to have intrauterine synechia, 16 patients $(26.7 \%)$ have submucous fibroids, 7 patients $(11.7 \%)$ have incomplete uterine septum and 6 patients $(10 \%)$ has bicornuate uterus.

The previously published data show large ranges of abnormal finding rates from one study to another $(7.2 \%$ to $64 \%){ }^{(12)}$. These differences could be explained by the type of range of distension medium and/or technique used, modifying the surgeon's perception of intrauterine filling defects. Results could also be influenced by the characteristics of the population: age of the population, hormonal status, ethnic factor, type of infertility (primary or secondary).

Preutthipan and Linasmita ${ }^{(13)}$ made a prospective comparative study between 
hysterosalpingography and SIS in the detection of intrauterine pathology in patients with infertility, included 336 patients undergoing both HSG and SIS. Intrauterine lesions were detected by SIS in 200 patients $(59.5 \%)$ and normal findings proved in 136 patients $(40.5 \%)$ of the whole sample. These results are nearly similar to the presented results in our study. The most common intrauterine finding of 336 patients on SIS were intrauterine adhesions (IUA), followed by endometrial polyps, and submucous myoma, 26 patients. In the current study the most common finding were endometrial polyp and intrauterine adhesions, followed by septate uterus then submucous myoma.

Another larger study done on one thousand consecutive infertile patients scheduled for in vitro fertilization underwent SIS, (38\%) of patients had abnormal intrauterine findings the commonest finding was endometrial polyps (32\%), the second common finding was intrauterine adhesions(3\%), and submucous fibroids (3\%), then other findings were; intrauterine septum $(0.5 \%)$ polypoid endometrium $(0.9 \%)$, septum $(0.5 \%)$ retained products of conception $(0.3 \%)$, and bicornuate uterus $(0.3 \%){ }^{(14)}$. This distribution of the abnormal findings is similar to that of our study where endometrial polyps were the commonest finding as well as intrauterine adhesions.

Lorusso et al. (15) evaluated the incidence of intrauterine pathologies in a population undergoing in vitro fertilization (IVF) by analyzing the SIS findings in 866 consecutive patients. About 514 cases (59.4\%) were considered normal; in 352 cases $(40.6 \%)$ various pathological conditions were found. These results are widely different from our results, this difference may be due to the smaller number of patients in the current study and the samples being designated to include cases of suspected uterine factor of infertility while Lorusso et al. ${ }^{(15)}$ included all cases of infertility.

In our study, with the use of HSG, the following findings were suspected; 9 cases showed normal uterine cavity (15\%), 51 cases $(85 \%)$ had different pathological findings. Out of the 9 normal cases, only 2 cases were confirmed by SIS (40\%), while the rest were false negative results $(60 \%)$. The findings which were missed were endometrial polypi (6 cases), and myoma (6 cases). This was interpreted in terms of sensitivity, specificity and accuracy of HSG in diagnosis of normal cases as $87.3 \%, 40 \%, 83.3 \%$ respectively. By HSG 14 cases of endometrial polyp were suspected its sensitivity $70 \%$, specificity $100 \%$ and accuracy $90 \%$ compared to SIS. In submucous myoma 10 cases were suspected, its sensitivity $62.5 \%$, specificity $100 \%$ and accuracy $90 \%$ compared to SIS. In septate uterus 7 cases were suspected, its sensitivity $100 \%$, its specificity $94.3 \%$ and accuracy $95 \%$ compared to SIS. In bicornuate uterus 6 cases were suspected, its sensitivity $100 \%$, its specificity $94.4 \%$ and accuracy $96.7 \%$ compared to SIS. In intrauterine synechia 6 cases were suspected, its sensitivity $100 \%$, specificity $94.4 \%$ and accuracy $95 \%$ compared to SIS. Shushan and Rojansky ${ }^{(11)}$ concluded that more than $1 / 3$ of the patients interpreted as normal following HSG were found to have a uterine abnormality after SIS, which might be a significant cause of reproductive failure. These women may be wrongly treated, or unnecessarily investigated, while their intrauterine lesion was missed.

Different pathological findings were suspected in 51 cases. Endometrial polypi were suspected in 14 cases $(23.3 \%)$. Sensitivity of HSG in diagnosis of polypi was $70 \%$, specificity 100\%, and accuracy $90 \%$. Intrauterine synechia were diagnosed in 9 patients (15\%). This made its specificity $94.4 \%$ while sensitivity was $100 \%$ and the accuracy was $95 \%$. In septate uterus 10 cases $(16.7 \%)$ were diagnosed by HSG, So sensitivity of HSG in diagnosis of septate uterus was $100 \%$, specificity $94.3 \%$ and accuracy $95 \% .10$ cases $(16.7 \%)$ were diagnosed as having submucous myomas by HSG. So specificity of HSG in diagnosis of myomas is $100 \%$, while sensitivity $62.5 \%$ and accuracy $90 \% .8$ cases of bicornuate uterus were suspected with HSG (13.3\%). This made its sensitivity $100 \%$, but specificity was $96.3 \%$, and accuracy $96.7 \%$.

These results can be compared to different previous studies which compared the findings of HSG to those of SIS.

Soares ${ }^{(16)}$ showed that HSG had a sensitivity of $58 \%$ and a positive-predictive value of $28.6 \%$ for polypoid lesions, this agrees with our study in the low sensitivity of HSG in diagnosis of endometrial polyps. The same study showed HSG to have a sensitivity of $44.4 \%$ for uterine malformations, and a sensitivity of $75 \%$ for the detection of 
intrauterine adhesions, which is much lower than our results ${ }^{(16)}$.

Shakya ${ }^{(17)}$ compared the accuracy of HSG in patients with infertility by a prospective comparative study done among 50 new cases of primary and secondary infertility. Results were out of 50 cases, HSG revealed normal findings in 49 cases $(98 \%)$ and SIS demonstrated normal uterine cavity in 44 of the cases $(88 \%),(10 \%$ false negative data in HSG). There was one abnormality (2\%) shown on HSG (subseptate uterus) which was confirmed at SIS. SIS demonstrated six cases (12\%) of intrauterine pathologies and these were endometrial polyps, subseptate uterus and submucous myoma ${ }^{(17)}$.

Gaglione et al. ${ }^{(18)}$ made a study over 70 patients, and showed that HSG gave a high number of false positive results: out of 33 patients with pathological findings by HSG, 13 were normal on SIS examination. Another fact emerging from the analysis is the presence of $18.9 \%$ false-negatives. Of 37 patients with normal HSG, seven had pathological SIS findings: four sessile submucosal myomas (1-2 $\mathrm{cm}$ in diameter, three on the anterior wall, one on the posterior wall), two fundal synechiae (< $33 \%$ of the uterine cavity) in patients with a history of abortion, and one endometritis confirmed by endometrial culture ${ }^{(\mathbf{1 8})}$.

Kumar et al. (19) also made a study comparing HSG and SIS over 60 infertile women. Statistical analyses showed specificity of HSG as $90 \%$ and false negative value as $40 \%$. High specificity indicates significant role for HSG as screening procedure but high false negative values show that intrauterine pathology can be missed by relying solely on HSG.

In our study the rate of false negative cases in HSG was $60 \%$ while there were no false positive cases.

This conflict between the result of our study and those by other authors is probably due to difference in technique and interpretation.

With the use of Doppler studies, the following results were obtained. 18 cases (30\%) were diagnosed as normal cases and 42 cases $(70 \%)$ were suspected to have different uterine pathologies. Out of the 18 normal cases, only 3 were confirmed by SIS, the rest were false negative values. The missed lesions included 1 case of intrauterine synechia, 1 case of septate uterus, 4 cases of submucous myoma and 11 cases of endometrial polyp. This can be interpreted in terms of sensitivity, specificity and accuracy of Doppler studies in diagnosis of normal cases as $72.7 \%, 60 \%$ and $71.7 \%$ respectively.

Out of the 42 cases with different pathological findings, endometrial polyp was suspected in 9 cases. This made its sensitivity $45 \%$, specificity $100 \%$ and accuracy $81.7 \%$ compared to SIS. In septate uterus 6 cases were suspected; it made its sensitivity $85.7 \%$, its specificity $100 \%$ and accuracy $98.3 \%$ compared to SIS in submucous myoma 12 cases were suspected its sensitivity $75 \%$, specificity $100 \%$ and accuracy $93.3 \%$ compared to SIS. In intrauterine synechia 5 cases were suspected; its sensitivity was $83.3 \%$ specificity $100 \%$ and accuracy $98.3 \%$ compared to SIS. In bicornuate uterus 6 cases were suspected; its sensitivity was $100 \%$, specificity $92.6 \%$ and accuracy $93.3 \%$ compared to SIS.

The introduction of PW Doppler US has provided a non-invasive mode of evaluation of uterine impedance and reveal physiological data, rather than anatomical information alone. There was significant correlation of uterine PI and biochemical markers of endometrial receptivity including endometrial histological dating (20).

Uterine artery impedance reflects the impedance of the whole uterine vascular bed, and may be affected by factors, such as fibroids, adenomyosis and contractility, not directly related to endometrial receptivity. Thus, endometrial blood flow may be a more specific indicator of receptivity, as shown in earlier studies ${ }^{(\mathbf{1 8})}$.

The quality and quantity of the spiral artery in the endometrium may be influenced by the uterine artery, but Doppler studies of uterine arteries do not reflect the actual blood flow to the endometrium, because the main portion of uterus is the myometrium, to which there are collateral circulations ${ }^{(\mathbf{2 1})}$.

Endometrial blood flow can now be evaluated noninvasively using color and power Doppler ultrasound. Power Doppler imaging is generally superior to color Doppler imaging for detecting low-velocity flows and visualizing small vessels ${ }^{(22)}$.

The combination of 2D ultrasound and power Doppler imaging has become a clinically feasible, fast, simple, and reproducible means of examining the blood supply to the whole endometrium and subendometrial, region ${ }^{(23)}$.

In addition to this it was reported that impedance of spiral artery blood flow in women 
with uterine factor of infertility was significantly higher than that of the fertile counterparts ${ }^{\text {(24). }}$.

In this study we observed that Doppler studies are better used to assess endometrial receptivity and a lot of studies revealed that ${ }^{(20)}$.

Steer et al. ${ }^{(24)}$ also suggested that decreased uterine perfusion might be a cause of unexplained infertility.

Using PW Doppler, they described increased impedance to blood flow within the uterine artery in women with no apparent cause for their subfertility.

Kurjak et al. ${ }^{(25)}$ also suggested that uterine factor of infertility may be, associated with aberrant uterine artery blood flow and intermittently absent end-diastolic flow.

It is somewhat surprising therefore that since these initial studies, the vast majority of imaging-based investigations have concentrated upon women undergoing ovarian stimulation as part of assisted reproduction treatment ${ }^{\text {(24). }}$.

There was significant correlation of uterine PI and biochemical markers of endometrial receptivity including endometrial histological dating ${ }^{(20)}$.

The measurement of impedance to uterine blood flow in IVF cycles has provided an indirect measure of endometrial receptivity (26).

In studies where endometrial and subendometrial blood flow were analysed by CD or PD US; absence of blood flow signal has been found to be associated with significantly low pregnancy rate or absence of pregnancy in IVF cycles ${ }^{(27)}$.

Infertility work up before IVF treatment does not include SIS or hysteroscope for every case, it includes HSG only which may be old or misinterpreted. Hysteroscope is done for cases with recurrent miscarriage, recurrent failed ICSI or suspected uterine pathology ${ }^{(19)}$.

Since Doppler assessment of subendometrial blood flow is becoming popular identifying, treatment and monitoring of IVF response, awareness of the relationship between abnormal findings and anatomic uterine abnormalities need to be known by every practitioner.

Abnormal uterine flow is not the standard modality to screen for uterine anatomic abnormalities, but it may raise suspicion and guide practitioners for more detailed investigations of abnormal uterine anatomy ${ }^{(22)}$
Our results showed that the spiral artery PI and RI were significantly increased in the uterine factor of positive cases. The range of RI in cases of infertility was $0.88-1.4$ and in negative cases RI range was $0.4-0.7$. We found the mean RI for the positive cases was $1.12+$ 0.16 , for negative cases was $0.58+0.06$ and the p-value was $<0.05$, which is statistically significant.

Battaglia et al. ${ }^{(28)}$ reported the highest pregnancy rate in the group with lower resistance to blood flow in the uterine spiral arteries. This finding coincides with our results as it revealed that the decrease in peripheral impedance in the uterine vasculature reflected by a low uterine artery PI was considered to be a consequence of increased blood flow and a sign of high tissue perfusion, and this might be an important prerequisite for successful IVF and ET cycle. In their study the best uterine receptivity was achieved in lower resistance group and no pregnancy were detected when $\mathrm{PI}>3.0$.

Kim et al. (29) examined the
relationship between endometrial,
subendometrial, uterine blood flow and
pregnancy after IUI, these studies found that
good endometrial blood flow is associated with
pregnancy, which is indicative of endometrial
receptivity and no such correlations existed
between subendometrial blood flow and
pregnancy ${ }^{(\mathbf{2 9})}$.

De Ziegler et al. ${ }^{(30)}$ also stated that Doppler US of endometrial and subendometrial regions does not contribute valid responses as to uterine receptivity and the chances for pregnancy.

Although our study is not involved in endometrial receptivity, IVF response and pregnancy rate, it is concerned with the identification of abnormal uterine pathology which may be a factor in IVF failure or recurrent abortion.

When spiral artery RI $>0.72$, PI $>1.6$ were present or there was absence of vascular signal in spiral artery, endometrium was considered to be non receptive, endometrial condition was suboptimal and implantation was unlikely to occur, so the patient was at high risk of ART treatment failure ${ }^{(21)}$.

Based on our findings, we can recommend that Doppler study of uterine hemodynamics should be considered in infertility work-up for better diagnostic capability and treatment modalities. Women 
with suboptimal uterine perfusion may be offered other diagnostic modalities for identifying abnormal uterine pathology.

PW US Doppler is technically difficult especially with tiny vessels like subendometrial signals and spiral arteries and operator dependant. This may be a limitation to the wide spread use of this technique ${ }^{(24)}$.

We therefore hypothesized that impaired blood flow could be an important contributing factor to infertility in women with no other relevant cause was present.

This finding suggests that abnormal endometrial perfusion may have a possible role in the pathogenesis of infertility. Doppler study of uterine hemodynamics should therefore be considered in infertility workup, and women with suboptimal uterine perfusion may be offered therapy aimed at improving uterine blood flow.

\section{CONCLUSION}

SIS is the gold standard of our study in assessment of uterine factor of infertility. Doppler studies show high false negative results compared to HSG and SIS. Some lesions missed from HSG and diagnosed by SIS and these missed lesions may play a role in infertility, so SIS is very important modality in diagnosis and assessment of uterine factor of infertility.

\section{REFERENCES}

1. Birch Petersen K, Maltesen T, Forman JL et al. (2017): The Fertility Assessment and Counseling Clinic - does the concept work? A prospective 2-year follow-up study of 519 women. Acta Obstet Gynecol Scand., 96(3): 313-325.

2. Vahdat M, Sariri E, Kashanian $M$ et al. (2016): comparative prospective study between hysterosalpingography and saline infusion sonohysterography in unexplained infertility. Med J Islam Repub Iran, 30: 352.

3. Armstrong SC, Showell M, Stewart EA et al. (2017): Baseline anatomical assessment of the uterus and ovaries in infertile women: a systematic review of the evidence on which assessment methods are the safest and most effective in terms of improving fertility outcomes. Hum Reprod Update, 23(5): 533-547.

4. Cogendez E, Eken MK, Bakal $\mathbf{N}$ et al. (2015): the role of transvaginal power Doppler ultrasound in differential diagnosis of intra uterine focal lesions. J Med Ultrason., 42(4): 533-40.

5. Jun WS, Lee KH and Koo K (2002): A straightforward algorithm for the quantification of power Doppler signals. Invest Radiol., 37(6): 343-8.

6. Raine-Fenning NJ, Campbell BK and Kendall NR (2004): Endometrial and subendometrial perfusion which impaired in women with unexplained subfertility, fen Reprod., 19 (II): 2605-14.

7. Kamel HS (2010): Management of the infertile couple: an evidence-based protocol. Reproductive Biology and Endocrinology, 6: 8-21.

8. Ombelet W, Cooke I, Dyer $S$ et al. (2008): Infertility and the provision of infertility medical services in developing countries. Hum Reprod Update, 14(6): 605-21.

9. Shiva M, Ahmadi F, Arabipoor A et al. (2018): Accuracy of two dimential ultrasound and hysterosalpingography and office hysteroscopy in uterine factor of infertility. Int J Fertil Steril., 11(4): 287292.

10. Grimbizis GF, Camus M, Tarlatzis BC et al. (2001): Clinical implications of uterine malformations and hysteroscopic treatment results. Hum Reprod., 7: 16174.

11. Shushan A and Rojansky N (1999): Should hysteroscopy be a part of the basic infertility workup? Hum Reprod., 14(8): 1923-1924.

12. Pansky M, Feingold $M$, Sagi $R$ et al. (2006): Diagnostic Hysteroscopy as a Primary Tool in a Basic Infertility Workup. Journal of the Society of Laparoendoscopic Surgeons, 10: 231-235.

13. Preutthipan $S$ and Linasmita V (2003): A prospective comparative study between hysterosalpingography and hysteroscopy in the detection of intrauterine pathology in patients with infertility, Journal of Obstetrics and Gynaecology Research, 29(1): 33-37.

14. Hinckley D, Amin $A$ and Milki $M$ (2004): $\quad 1000 \quad$ Office-Based Hysteroscopies Prior to In Vitro Fertilization: Feasibility and Findings, JSLS., 8(2): 103-107.

15. Lorusso F, Ceci O, Bettocchi $S$ et al. (2008): Office hysteroscopy in an in vitro 
fertilization program. Gynecol Endocrinol., 24(8): 465-9.

16. Soares SR (2000): Diagnostic accuracy of sonohysterography, Fertility and sterility, 73 (2): 406-411

17. Shakya B (2009): Paropakar Maternity and Women's Hospital, Thapathali, Kathmandu, Nepal. Hysterosalpingography Vs Hysteroscopy in the Detection of Intrauterine Pathology in Infertility, J Nepal Health Res Counc., 7(14): 6-9.

18. Gaglione $R$ (1996): A comparison of hysteroscopy and hysterosalpingography /International Journal of Gynecology \& Obstetrics, 52: 151-153.

19. Kumar V, Awasthi S and Gokhale I (2003): Assessment of Uterine Factor in Infertile Women:Hysterosalpingography vs Hysteroscopy MJAFI., 60: 39-41.

20. Steer CV, Tan SL and Dillon D (1995): Vaginal color Doppler assessment of uterine artery impedance correlates with immunohistochemical markers of endometrial receptivity required for the implantation of an embryo. Fertil Steril., 63(1): 101-8.

21. Ng EH, Chan CC and Tang OS (2006): Relationship between uterine blood flow and endometrial and subendometrial blood flows during stimulated and natural cycles. Fertil Steril., 85(3): 7-21.

22. Guerriero $S$, Ajossa $S$ and Lai MP (1999): Clinical applications of colour Doppler energy imaging in the female reproductive tract and pregnancy. Hum Reprod Update, 5(5): 515-29.

23. Merce LT, Barco MJ and Bau S (2008): Are endometrial parameters by threedimensional ultrasound and power
Doppler angiography related to in vitro fertilization/embryo transfer outcome? Fertil Steril., 89(1): 111-7.

24. Steer CV, Tan SL and Mason BA (1994): Midluteal-phase vaginal color Doppler assessment of uterine artery impedance in a subfertile population. Fertil Steril., 61(1): 538.

25. Kurjak A, Kupesic-Urek $S$ and Schulman H (1991): Transvaginal color flow Doppler in the assessment of ovarian and uterine blood flow in infertile women. Fertil Steril., 56(5): 870-3.

26. Dechaud $H$, Bessueille $E$ and Bousquet PJ (2008): Optimal timing of ultrasonographic and Doppler evaluation of uterine receptivity to implantation. Reprod Biomed Online, 16(3): 368-75.

27. Chien LW, Au HK and Chen PL (2002): Assessment of uterine receptivity by the endometrial-subendometrial blood flow distribution pattern in women undergoing in vitro fertilization-embryo transfer. Fertil Steril., 78(2): 245-51.

28. Battaglia $\mathbf{C}$, Larocca $\mathbf{E}$ and Lanzani A (1990): Doppler ultrasound studies of the uterine arteries in spontaneous and IVF stimulated ovarian cycles. Gynecol Endocrinol., 4(4): 245-50.

29. Kim A, Han JE and Yoon TK (2010): Relationship between endometrial and subendometrial blood flow measured by three-dimensional power Doppler ultrasound and pregnancy after intrauterine insemination. Fertil Steril., 94(2): 747-52.

30. De Ziegler D, Pirtea $P$, Galliano $D$ et al. (2016): optimum uterine anatomy and physiology necessary for normal implantation Fertil Steril., 105(4): 844-54. 